\title{
Age-related differences in white matter microstructure: Region-specific patterns of diffusivity
}

\author{
A.Z. Burzynska ${ }^{\text {a,* }}$, C. Preuschhof ${ }^{\text {a }}$, L. Bäckman ${ }^{\text {a,b }}$, L. Nyberg ${ }^{\text {c }}$, S.-C. Li ${ }^{\text {a }}$, U. Lindenberger ${ }^{\text {a }}$, H.R. Heekeren ${ }^{\text {a,d }}$ \\ a Max Planck Institute for Human Development, Lentzeallee 94, D-14195 Berlin, Germany \\ ${ }^{b}$ Aging Research Center, Karolinska Institute, Gävlegatan 16, SE-11330, Stockholm, Sweden \\ c Department of Radiation Sciences (Diagnostic Radiology) and Integrative Medical Biology (Physiology Section), Umeå University, S-901 87 Umeå, Sweden \\ ${ }^{\mathrm{d}}$ Max Planck Institute for Human Cognitive and Brain Sciences, Stephanstraße 1a, 04103 Leipzig, Germany
}

\section{A R T I C L E I N F O}

\section{Article history:}

Received 3 March 2009

Revised 15 August 2009

Accepted 21 September 2009

Available online 25 September 2009

Keywords:

Axial diffusivity

Fractional anisotropy

Mean diffusivity

Radial diffusivity

Tract-based spatial statistics

\begin{abstract}
A B S T R A C T
We collected MRI diffusion tensor imaging data from 80 younger (20-32 years) and 63 older (60-71 years) healthy adults. Tract-based spatial statistics (TBSS) analysis revealed that white matter integrity, as indicated by decreased fractional anisotropy (FA), was disrupted in numerous structures in older compared to younger adults. These regions displayed five distinct region-specific patterns of age-related differences in other diffusivity properties: (1) increases in both radial and mean diffusivity; (2) increases in radial diffusivity; (3) no differences in parameters other than FA; (4) a decrease in axial and an increase in radial diffusivity; and (5) a decrease in axial and mean diffusivity. These patterns suggest different biological underpinnings of agerelated decline in FA, such as demyelination, Wallerian degeneration, gliosis, and severe fiber loss, and may represent stages in a cascade of age-related degeneration in white matter microstructure. This first simultaneous description of age-related differences in FA, mean, axial, and radial diffusivity requires histological and functional validation as well as analyses of intermediate age groups and longitudinal samples.
\end{abstract}

(C) 2009 Elsevier Inc. All rights reserved.

\section{Introduction}

The human brain displays significant structural and functional changes across adulthood (Raz and Rodrigue, 2006). The volume of white matter (WM), as measured by in vivo magnetic resonance imaging (MRI), continues to increase until the fourth decade of life and is associated with ongoing myelination (Bartzokis, 2004; Courchesne et al., 2000; Ge et al., 2002; Giedd, 1999). The subsequent decrease in WM volume accelerates in late adulthood (Courchesne et al., 2000; Raz et al., 2005) and coincides with a smaller number and shorter length of myelinated fibers, myelin pallor, and axonal loss as shown in post-mortem histological studies (Aboitiz et al., 1996; Marner et al., 2003; Meier-Ruge et al., 1992; Tang and Nyengaard, 1997).

Due to limitations of histological studies (restricted possibility of sample selection, necessity of tissue fixation, and resulting artifacts), none of these previous studies addressed regional specificity of the aforementioned microstructural alterations in aging. In contrast, in vivo structural MRI studies allow identifying regional differences in WM volume loss. They do, however, not provide much insight into the mechanisms responsible for these aging-related WM changes. In this

\footnotetext{
* Corresponding author. Fax: +49 308249939.

E-mail address: burzynska@mpib-berlin.mpg.de (A.Z. Burzynska).
}

study, we therefore employed diffusion tensor imaging (DTI), which combines some of the benefits of both histological and volumetric approaches in studying WM.

By quantifying the magnitude and directionality of diffusion of water within a tissue, DTI allows inferences about WM microstructure in vivo (Pierpaoli and Basser, 1996; Pierpaoli et al., 1996). Investigating DTI-derived parameters may help to elucidate the mechanisms underlying age-related WM changes because different parameters reflect distinct aspects of WM microstructure. The eigenvalues of the diffusion tensor are obtained through diffusion tensor diagonalization (Basser and Pierpaoli, 1996, 1998; Pierpaoli and Basser, 1996). The first eigenvalue is referred to as axial diffusivity (AD, diffusion parallel to the axon fibers; Basser, 1995; Song et al., 2002), whereas the average of the second and third eigenvalues is termed radial diffusivity (RD, diffusivity perpendicular to the axonal fibers; Basser, 1995; Song et al., 2002). Lowered AD reflects axon injury both in ischemic (Song et al., 2003, 2005) and chemically induced (Sun et al., 2006) WM lesions. Increases in RD have been linked to incomplete myelination in shiverer mice (Song et al., 2002), drug-induced demyelination (Song et al., 2005), and loss of myelin following axon injury (Song et al., 2003, 2005). Fractional anisotropy (FA), a measure of the directional dependence of diffusion (Basser, 1995), reflects fiber density and coherence within a voxel (Beaulieu, 2002). FA values range between 0 (isotropic diffusion) and 1 (perfectly anisotropic diffusion). Lowered FA has been observed in various conditions in 
which loss of fiber integrity occurs (Beaulieu, 2002), such as multiple sclerosis (Rovaris et al., 2005) and Alzheimer's disease (Medina et al., 2006). Mean diffusivity (MD) is a mean of all three eigenvalues of the diffusion tensor and reflects the magnitude of water diffusion within a voxel, which depends on the density of physical obstructions such as membranes and the distribution of water molecules between different cellular compartments (Beaulieu, 2002; Sen and Basser, 2005). Increased MD was observed in conditions of reduced membrane density (Sen and Basser, 2005) such as tissue degeneration after injury (Beaulieu, 2002; Beaulieu et al., 1996; Concha et al., 2006). Thus, comparing the values of $\mathrm{FA}, \mathrm{MD}, \mathrm{RD}$, and $\mathrm{AD}$ between younger and older adults allows inferring potential age-related microstructural mechanisms underlying the observed differences in diffusivity properties.

WM diffusivity changes in late adulthood-FA decreases and MD increases (Minati et al., 2007)-likely reflect microstructural alterations such as an increase in brain water content, demyelination, disruption of axon structure, and overall rarefaction of fibers (Minati et al., 2007). To obtain a comprehensive picture of differences in different elements of WM microstructure, however, it is necessary to consider conjointly all measures derived from the diffusion tensor (Assaf and Pasternak, 2008). This has not been done systematically in previous research; few studies have reported eigenvalues for selected WM structures (Abe et al., 2002; Bhagat and Beaulieu, 2004; Hsu et al., 2008; Ota et al., 2006; Stadlbauer et al., 2008; Sullivan et al., 2006b), and only one tractography-based study reported the values of MD, RD, and AD for all main cerebral WM tracts in adults between 20 and 81 years of age (Sullivan et al., in press). The diffusivities were, however, not analyzed conjointly and studying a mean value for the whole tract does not use the localized information of diffusivity properties obtained for each voxel given that the age effect on diffusivity properties may not be uniform along a WM tract.

Investigating the direct relationship between age effects on different diffusivity parameters could provide more insight into the mechanisms of WM aging. Zhang et al. (in press) identified WM regions with simultaneous age-related variations in FA and $\mathrm{MD}$, as well as regions showing FA differences without a significant MD difference and vice versa. In a separate analysis, they showed that agerelated increases in $\mathrm{RD}$ were of greater magnitude than the corresponding increases in AD (Zhang et al., in press). Still, the direct relationship between these four DTI measures remains to be evaluated. Due to the lack of simultaneous analysis of FA, RD, and $A D$, Zhang's conclusion that increased RD mostly explains the FA reductions is not justified. By analyzing RD and $\mathrm{AD}$ separately from FA and $\mathrm{MD}$ it is also impossible to distinguish between diffusivity patterns characteristic of Wallerian degeneration (FA and AD decrease, RD increase and no net difference in MD, Pierpaoli et al., 2001 ) and patterns characteristic of other mechanisms (FA decrease and no difference in other diffusivities).

Zhang et al. (in press) applied voxel-based morphometry (VBM) analysis, an approach originally designed for assessing changes in grey matter density. This approach includes smoothing of the diffusivity maps, a step that is generally not recommended for DTI data (Jones et al., 2005). In addition, this approach does not allow precise tractrelated localization of the observations. Tract-based spatial statistics (TBSS; Smith et al., 2006) is an alternative approach for analyzing differences in WM. TBSS brings together the strengths of whole-brain VBM methods and localized region-of-interest (ROI) analyses. TBSS circumvents the problem of cross-subject alignment and contamination due to differences in brain morphology by sampling the centerof-tract voxel value in individual space. It, therefore, enables reliable detection of localized differences in diffusivity parameters in all major WM tracts without the necessity of smoothing (Smith et al., 2006). The applicability of TBSS to aging studies has been demonstrated in two recent studies. In a study comparing 8 younger and 22 older participants, Damoiseaux et al. (2009) reported age-related FA decreases in the frontal, parietal, and temporal lobes, corpus callosum, and the internal capsule. In a large sample of adults above the age of $60(n=832)$, Vernooij et al. (2008) examined the relationships between DTI parameters and macrostructural differences, such as overall brain atrophy and lesion volumes.

In this study, we investigated differences in WM diffusivity properties between healthy adults in their third and seventh decade of life. Specifically, we addressed the following questions: What is the voxelwise pattern of simultaneous differences in FA, MD, AD, and RD in the whole WM from early to late adulthood? Are the patterns of age-related differences in diffusivity parameters not only region- but also tract-specific? What is the neurobiological meaning of these patterns and are they meaningful in view of extant histological knowledge?

We analyzed FA, MD, RD, and $\mathrm{AD}$ in all major WM tracts of the cerebrum using TBSS. Next, we investigated, on the voxel level, the spatial distribution of age-related differences in $\mathrm{AD}, \mathrm{RD}$, and $\mathrm{MD}$ within those regions showing age-related FA decreases. This approach allows for a more specific interpretation of age-related decreases in FA as opposed to considering $\mathrm{FA}$ and $\mathrm{MD}$ or $\mathrm{AD}$ and $\mathrm{RD}$ alone. We hypothesized that WM tracts and their subregions would differ with respect to age-related differences in diffusivity properties. For instance, we predicted that in the regions most susceptible to aging, such as the frontal lobes, irreversible loss of myelinated fibers would result in a decrease in FA and a general increase in diffusivity. In other regions, axonal injury may result in a decrease in $A D$ and demyelination may cause an increase in RD. In some regions, age effects may be represented mainly by glial infiltration resulting in lowered FA and MD. Taken together, the analysis of region-specific patterns in diffusivity properties may reveal spatial specificity of differential age-related differences in WM microstructure.

\section{Materials and methods}

\section{Participants}

The participants were 80 younger adults ( 35 women) between 20 and 32 years of age $(M=25.7 \pm 3.2)$ and 63 older adults ( 29 women) between 60 and 71 years of age $(M=64.8 \pm 2.9)$, all of whom had at least 8 years of education, were right-handed, and had no history of psychiatric or neurological disease. For 58 of 63 older adults, we had information about their vascular risk factors: 25 (43\%) reported arterial hypertension (defined as blood pressure constantly above $140 / 90 \mathrm{~mm} \mathrm{Hg}$ ), 11 of the 25 hypertensive participants took antihypertensive medication, 5 (8.6\%) had type 2 diabetes, all of which took medication but only one was insulin-dependent, and 6 (10.4\%) reported hypercholesterolemia ( 2 of which were taking medications). Older adults showed a decline in fluid abilities (perceptual speed), as measured by digit-symbol substitution $\left(M_{\text {younger }}=63.1 \pm 10.9\right.$, $M_{\text {older }}=47.8 \pm 12.7, p<0.001$ ), but higher crystallized abilities (verbal knowledge), as measured by Spot-a-Word task ( $M_{\text {younger }}=18.3 \pm 5.3$, $M_{\text {older }}=22.2 \pm 5.8, p<0.001$ ), which is typical for an aged population (Li et al., 2004).

The ethics committee of the Charité University Medicine Berlin and the Max Planck Institute for Human Development approved the study, and written informed consent was obtained from all participants prior to the examination.

\section{MR acquisition}

Diffusion-weighted and fluid-attenuated inversion recovery (FLAIR) images were acquired on a $1.5 \mathrm{~T}$ Siemens Sonata with $40 \mathrm{mT} / \mathrm{m}$ gradients and $200 \mathrm{~T} / \mathrm{m} / \mathrm{sec}$ slew rates (Siemens, Erlangen, Germany). All images were obtained parallel to the anterior-posterior commissure plane with no interslice gap. FLAIR images consisted of 24 5 -mm-thick slices with an in-plane resolution of $1.3 \times 0.9 \mathrm{~mm}$ 
$(240 \times 256$ matrix, TR/TE/TI $=7500 / 118 / 2200 \mathrm{~ms})$. Eddy currentinduced image distortions were minimized by using a twicerefocused spin echo single-shot Echo Planar Imaging sequence to acquire DTI data (Reese et al., 2003), with TR/TE $=8500 / 96 \mathrm{~ms}$, $128 \times 128$ matrix, $2.5 \mathrm{~mm}^{2}$ in-plane resolution, and receiver bandwidth of $1502 \mathrm{~Hz}$, comprising 52 2.5-mm-thick slices. The protocol consisted of a set of 12 non-collinear diffusion-weighted acquisitions with $b$-value $=1000 \mathrm{~s} / \mathrm{mm}^{2}$ and a T2-weighted, $b$-value $=0 \mathrm{~s} / \mathrm{mm}^{2}$ acquisition, each repeated four times.

\section{Assessment of white matter hyperintensity (WMH) burden}

A.Z.B. evaluated the presence of age-related WMHs on FLAIR images using the rating scale described by Wahlund et al. (2001). The presence of moderate WMHs is typical for a normally aged population (Baloh and Vinters, 1995). A neuroradiologist examined the structural images of participants with WMH grade 2 and classified them within the normal range for their age.

We estimated WMH volume using a semi-automated procedure based on FMRIB's Automated Segmentation Tool (FAST 4.1, Zhang et al. 2001). The procedure included: (a) removal of the skull and non-brain tissue using the Brain Extraction Tool (Smith, 2002), (b) segmentation of the image into three tissue types (grey and white matter, cerebrospinal fluid (CSF)), and (c) manual masking of WMH regions within the CSF segmentation (done by A.Z.B.). WMH volumes are given in number of in-plane voxels. All younger and 19 older participants had grade 0 (no lesions), 29 older adults had grade 1 (a single to several focal WM lesions, $M_{\mathrm{WMH}}$ volume $=962 \pm 697$ voxels, range 117-2,615 voxels), 15 older participants had grade 2 (beginning confluence of lesions, $M_{\mathrm{WMH}}$ volume $=7414 \pm 5,496$ voxels, range $3094-23,338$ voxels). The difference in WMH volume between grade 1 and grade 2 was significant ( $p<0.001$, independent-samples $t$-test). We found no relationship between WMH volume and age within older adults ( $r=0.20, p=0.11$ ), but considering the whole sample, we found the expected correlation between WMH volume and age $(r=0.40, p<0.001)$. For 50 of 63 older adults from whom we had the blood pressure measures, we found no correlation between $\mathrm{WMH}$ volume and blood pressure $\left(r_{\mathrm{WMH}}\right.$ volume-systolic $=$ $-0.03, r_{\mathrm{WMH}}$ volume-diastolic $=0.07, r_{\mathrm{WMH}}$ volume-MAP $\left.=0.03\right)$.

\section{Analysis of DTI data}

DTI data were processed using the FSL 4.1 Diffusion Toolbox (FDT: http://www.fmrib.ox.ac.uk/fsl/) in a standard multistep procedure, including: (a) motion and eddy current correction, (b) removal of the skull and non-brain tissue using the Brain Extraction Tool (Smith, 2002), and (c) voxel-by-voxel calculation of the diffusion tensors. Using the diffusion tensor information, $\mathrm{FA}, \mathrm{MD}$, and $\mathrm{AD}$ maps were computed using DTIFit within the FDT. RD was calculated as the mean of the second and third eigenvalues (Song et al., 2002).

\section{Tract-based spatial statistics}

Voxelwise statistical analysis of the diffusion maps was carried out using TBSS v1.2 (Smith et al., 2006; Smith et al., 2007), a toolbox within FSL (Smith et al., 2004), and included: (a) nonlinear alignment of each participant's FA volume to the $1 \times 1 \times 1 \mathrm{~mm}^{3}$ standard Montreal Neurological Institute (MNI152) space via the FMRIB58_FA template using the FMRIB's Nonlinear Registration Tool (FNIRT, Rueckert et al., 1999; www.doc.ic.ac.uk/ dr/software), (b) calculation of the mean of all aligned FA images, (c) creation of the WM "skeleton" (a representation of WM tracts common to all subjects) by perpendicular non-maximum-suppression of the mean FA image and setting the FA threshold to 0.25 , (d) perpendicular projection of the highest FA value (local center-of-tract) onto the skeleton, separately for each subject, and (e) feeding the data into a voxelwise permutation-based (5000 permutations) inference (Nichols and
Holmes, 2002). Voxels thresholded at $t>3$, corrected for multiple comparisons with the cluster significance threshold of $p<0.01$ (2tailed), were considered significant.

\section{Pattern analysis}

Non-FA parameter analysis was done as follows: the transformation matrix from step (a) and the projection vectors from step (d) were used to align the values of MD, RD, and AD into the MNI152 space and to project them onto the skeleton. In the final step, the resulting images were subjected to the same statistical analysis as the FA data. The resulting maps of $p$ values were masked to only include the voxels showing significant age-related differences in FA. We multiplied the masked $p$ value maps of FA and the other parameters to create conjunction images (i.e., we performed a logical AND operation between the thresholded FA map and the other parameter maps). After setting the appropriate significance thresholds $(p<0.01,2$ tailed), we evaluated the spatial overlap of the conjunction images.

\section{Results}

\section{Age-related differences in $F A$}

For both younger and older adults, FA values differed among brain regions. For more details on regional specificity of FA values on the TBSS skeleton, see Supplementary Materials.

We performed voxelwise comparison of age-related differences in FA values between younger and older adults. FA was lower in older than in younger adults $(p<0.01)$ in numerous WM regions, which included: the anterior, superior and posterior corona radiata, WM of the superior, inferior, middle, frontal and straight gyri, WM of the precuneus and superior parietal lobule, parts of the cingulum (mainly dorsal), the body and the column of the fornix, the forceps minor and major, the external capsule, the internal capsule, the sagittal stratum, and the parahippocampal WM (See Fig. S-2, Supplementary materials). In the anterior limb of the internal capsule, posterior part of the dorsal cingulum, the body and the splenium of the corpus callosum, the forceps major and white matter within the occipital lobe, and part of the inferior cerebellar peduncles, the age-related difference in FA was not significant after accounting for WMH burden (Fig. S-4, Supplementary materials). We observed no age-related FA increases. For the comparison of the mean $\mathrm{FA}, \mathrm{MD}, \mathrm{RD}$, and $\mathrm{AD}$ values from the whole WM skeleton, see Supplementary Materials.

Patterns of differences in axial, radial, and mean diffusivity in regions showing lowered FA with aging

Within WM structures showing an age-related reduction in FA, conjunction analyses revealed a complex pattern of significant increases in $\mathrm{RD}, \mathrm{MD}$ and $\mathrm{AD}$, as well as decreases in $\mathrm{AD}$ and MD. In the majority of WM regions, there was a single dominant pattern of the overlapping age-related diffusivity differences. Altogether we distinguished five main patterns: (1) An increase in both RD and MD was predominant in the genu of the corpus callosum, the middle frontal gyrus, the external capsule, the anterior limb of the internal capsule, the anterior part of the dorsal cingulum, and in the sagittal stratum. In some of these regions, AD was also increased with age, particularly in the body of the corpus callosum (Fig. 1-1); (2) Other regions such as the superior corona radiata, forceps minor and large parts of the dorsal posterior cingulum (Fig. 1-2) predominantly showed an increase in RD; (3) Several structures showed a reduction in FA, without significant differences in the other parameters: the ventral part of the middle frontal gyrus, forceps minor, and the straight gyrus (Fig. 1-3); (4) In the crus of the fornix and retrolenticular part of the internal capsule, an increase in RD was accompanied by a decrease in AD (Fig. 1-4); (5) In the WM of the midbrain and cerebellar peduncles, there was a significant age-related 

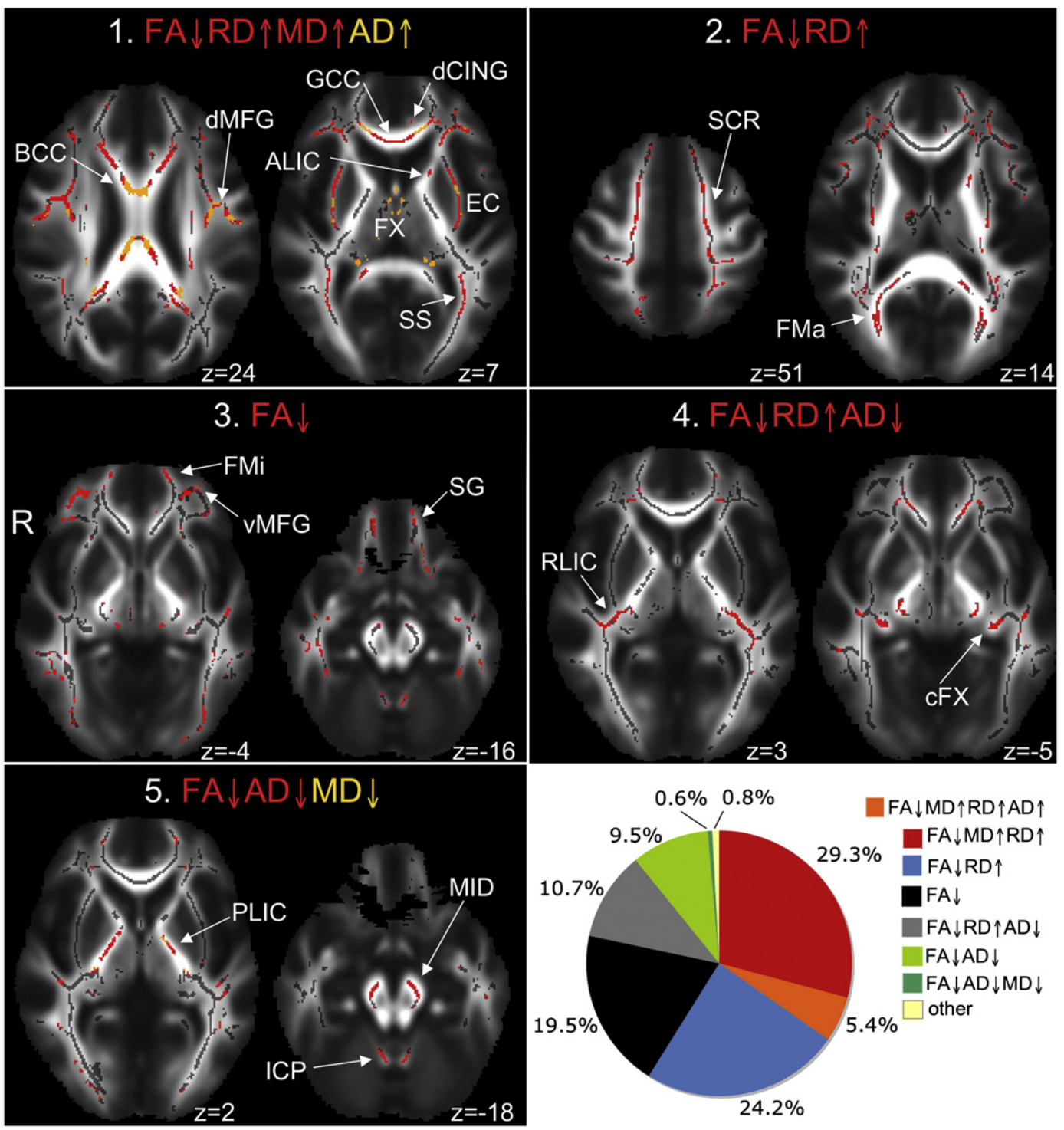

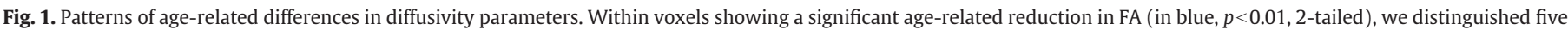

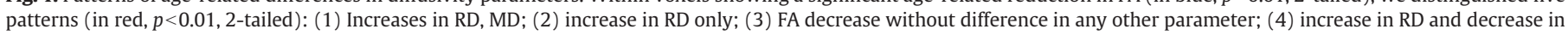

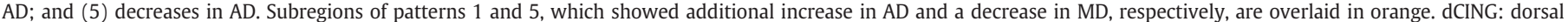

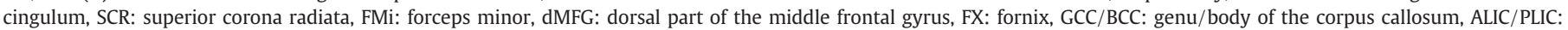

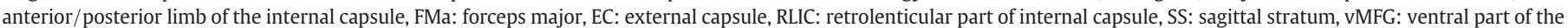

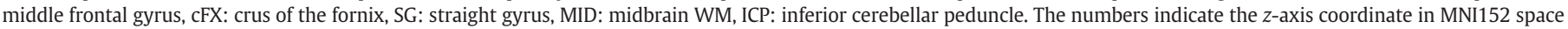

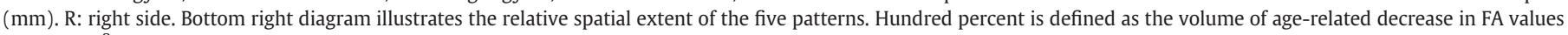
$55,973 \mathrm{~mm}^{2}$.

decrease in $\mathrm{AD}$, which was accompanied by a decrease in MD in the posterior limb of the internal capsule (Fig. 1-5). Few scattered voxels showed a decrease in FA and AD and an increase in RD and MD. These voxels were classified as "others" in the diagram in Fig. 1 and they are not considered in the discussion. Within voxels showing lower FA in older participants, we observed no decrease in RD. Table 1 summarizes the spatial localization of these patterns.

A simultaneous increase in RD and MD was the dominant pattern (34.7\%), whereas a decrease in $\mathrm{AD}$ and an increase in RD occupied the smallest area (10.1\% and $10.7 \%$, respectively) of the total volume showing an age-related reduction in FA (Fig. 1).

Given that the eigenvalues are by definition orthogonal, while their derivatives (such as FA and MD) may not be, we performed a post-hoc analysis to investigate the dependency between FA and MD within ROIs defined within each of the five patterns described above. The correlations between FA and MD were as follows: genu of the corpus callosum (pattern $1, r=-0.87, p<0.001$ ), superior corona radiata (pattern 2, $r=-0.61, p<0.001$ ), forceps minor (pattern 3, $r=-0.63, p<.001$ ), crus of the fornix (pattern $4, r=-0.15$, $p=0.08$ ), and the inferior cerebral peduncle (pattern $5, r=0.14$, $p=0.10$ ). These results show that (a) the dependency between FA and MD is region-specific and (b) FA and MD are not necessarily correlated.

\section{Discussion}

The aim of this study was to investigate the whole-brain pattern of age-related differences in all diffusivity parameters and to explore their regional specificity. For the analysis of FA, MD, RD, and $A D$ values, we used TBSS, which accounts for morphological differences that can be observed between healthy adults in the third and seventh decade of life. In addition to the previously reported anteroposterior gradient of age-related FA decrease in the corpus callosum (Head et al., 2004; Salat et al., 2005; Sullivan et al., 2001), we detected FA 
Table 1

Summary of spatial colocalization of age-related differences in RD, AD, and MD within regions showing an age-related reduction in FA ( $t>3, p<0.01,2$-tailed)

\begin{tabular}{|c|c|c|c|c|c|c|c|c|}
\hline Pattern & Anatomic location & $\mathrm{FA} \downarrow \mathrm{RD} \uparrow \mathrm{MD} \uparrow$ & $\mathrm{FA} \downarrow \mathrm{RD} \uparrow \mathrm{MD} \uparrow \mathrm{AD} \uparrow$ & $\mathrm{FA} \downarrow \mathrm{RD} \uparrow$ & $\mathrm{FA} \downarrow$ & $\mathrm{FA} \downarrow \mathrm{RD} \uparrow \mathrm{AD} \downarrow$ & $\mathrm{FA} \downarrow \mathrm{AD} \downarrow$ & $\mathrm{FA} \downarrow \mathrm{AD} \downarrow \mathrm{MD} \downarrow$ \\
\hline \multirow[t]{8}{*}{1} & Genu of the corpus callosum & a & $\mathrm{b}$ & c & - & c & - & - \\
\hline & Anterior limb of internal capsule & a & - & c & c & - & - & - \\
\hline & Dorsal middle frontal gyrus & a & b & b & c & - & - & - \\
\hline & External capsule & a & b & c & - & - & - & - \\
\hline & Sagittal stratum & a & - & b & $\mathrm{b}$ & - & - & - \\
\hline & Anterior dorsal cingulum & a & b & b & - & - & - & - \\
\hline & Body of the corpus callosum & b & a & c & - & - & - & - \\
\hline & Fornix & c & a & - & - & - & - & - \\
\hline \multirow[t]{3}{*}{2} & Posterior dorsal cingulum & c & c & $\mathrm{b}$ & b & - & - & - \\
\hline & Forceps major & - & - & a & c & c & c & - \\
\hline & Superior corona radiata & b & - & a & - & b & - & - \\
\hline \multirow[t]{3}{*}{3} & Forceps minor & c & - & c & a & c & c & - \\
\hline & Ventral middle frontal gyrus & c & - & c & a & - & - & - \\
\hline & WM of the straight gyrus & b & - & b & a & c & - & - \\
\hline \multirow[t]{2}{*}{4} & Retrolenticular internal capsule & - & - & - & - & a & c & - \\
\hline & Crus of the fornix & c & - & c & c & a & c & - \\
\hline \multirow[t]{3}{*}{5} & Posterior limb of internal capsule & - & - & - & - & c & a & b \\
\hline & Midbrain WM & c & - & c & c & c & a & - \\
\hline & Cerebellar peduncles & - & - & - & b & - & a & b \\
\hline
\end{tabular}

${ }^{a}$ Dominant pattern.

b Present.

${ }^{\mathrm{c}}$ Single voxels.

- Pattern absent.

reduction in multiple locations throughout the whole cerebral WM, particularly in regions containing associative connections of the frontal cortex. Within the structures showing lower fiber integrity in older adults (as measured by FA), WM regions showed distinct patterns of increases in RD and both decreases and increases in MD and $A D$, whereas other regions revealed differences in FA only. These diffusivity patterns suggest that the age-related microstructural alterations in WM differ by region. Here we discuss possible biological underpinnings of the observed diffusivity patterns.

\section{TBSS revealed age-related differences in FA throughout cerebral WM}

The whole-WM TBSS analysis showed that higher adult age was associated with lower FA values in as many as 53\% of the center-oftract voxels considered in the analysis. For most of these regions, there is some previous evidence for age-related FA decreases. These include the anterior parts of the corpus callosum (Camara et al., 2007; Head et al., 2004; Hsu et al., 2008; Hugenschmidt et al., 2008; Ota et al., 2006; Salat et al., 2005; Sullivan et al., 2001, 2006a; Yoon et al., 2008), the external capsule (Ardekani et al., 2007; Bhagat and Beaulieu, 2004; Hugenschmidt et al., 2008), the prefrontal WM (Bucur et al., 2008; Camara et al., 2007; Grieve et al., 2007; Hsu et al., 2008; Hugenschmidt et al., 2008; Madden et al., 2004; Pfefferbaum et al., 2000a; Salat et al., 2005), the sagittal stratum (Pfefferbaum et al., 2005), the posterior thalamic radiations (Hugenschmidt et al., 2008), the internal capsule (Ardekani et al., 2007; Bhagat and Beaulieu, 2004; Hsu et al., 2008; Salat et al., 2005; Yoon et al., 2008), the corona radiata and the adjacent WM (Bucur et al., 2008; Camara et al., 2007; Hugenschmidt et al., 2008; Pfefferbaum et al., 2000a; Sullivan et al., 2001; Yoon et al., 2008), the cingulum (Ardekani et al., 2007; Bucur et al., 2008; Sullivan et al., in press; Yoon et al., 2008), and the fornix (Stadlbauer et al., 2008).

To the best of our knowledge, there are only two studies that assessed FA differences in the direct vicinity of the hippocampus: one ROI-based study (Yoon et al., 2008) and one TBSS study (Vernooij et al., 2008), both of which found a bilateral FA reduction in old age. In agreement with these studies, we showed that FA is decreased in old age in the direct vicinity of the hippocampal formation, namely in the crus of the fornix.

Medial temporal lobe structures including the hippocampus are critical to episodic memory functioning (Eichenbaum and Cohen, 2001). Hippocampal alterations might, therefore, be an important factor underlying age-related deficits in episodic memory. This is supported by findings of a bilateral reduction in hippocampal activation during episodic retrieval in older adults (Cabeza et al., 2004; Daselaar et al., 2006). The present findings suggest that the hippocampal component of age-related episodic memory deficits might not only be due to grey matter shrinkage (Raz et al., 2005), but also reflect alterations in hippocampal WM connections (see also Persson et al., 2006).

\section{Connection-wise anteroposterior gradient of age-related reduction in FA}

The anterior parts of the corpus callosum showed greater agerelated decreases in FA values than the posterior parts, thereby confirming the proposed age-related anteroposterior gradient of FA decline in the corpus callosum (Abe et al., 2002; Camara et al., 2007; Hsu et al., 2008; Hugenschmidt et al., 2008; Ota et al., 2006; Pfefferbaum et al., 2005, 2000b; Salat et al., 2005; Sullivan et al., 2001, 2006b; Sullivan and Pfefferbaum, 2006). This gradient was, however, less obvious in the whole WM, as age-related FA reductions were present within multiple, widely distributed regions of the cerebrum (similar to Damoiseaux et al., 2009; Vernooij et al., 2008) and in the cerebellar WM. Still, from a systemic perspective, it is apparent that the decrease in FA primarily affects the connections of the frontal lobe, which is in agreement with extant patterns of agerelated cognitive deficits (Paxton et al., 2008).

FA reductions in the anterior parts of the corpus callosum suggest a disruption of the interhemispheric communication at the level of prefrontal, premotor, and supplementary motor cortices (Hofer and Frahm, 2006). FA decreases in the anterior limb of the internal capsule and the anterior corona radiata, containing both commissural fibers of the corpus callosum and the anterior thalamic radiations, suggest a disruption in the thalamocortical connections of the frontal lobe (Schmahmann and Pandya, 2006). Similarly, age-related FA reductions in the WM of the straight, inferior and middle frontal gyri suggest decreased integrity of the local connections within the frontal lobe, in particular in the lateral prefrontal cortex. FA decreases within the ventral part of the middle frontal gyrus, in the ventral parts of the external capsule, and in the WM of the temporal pole indicate agerelated alterations of the uncinate fasciculus, which contains reciprocal connections between the ventrolateral prefrontal cortex and anterior parts of the temporal lobe, including the amygdala (Schmahmann and Pandya, 2006). The decreased FA in the external 
capsule may also affect fibers connecting the ventral and medial prefrontal cortex to the striatum. FA decreases within the dorsal parts of the middle frontal gyri (dorsolateral prefrontal cortex), in the dorsal parts of the external capsule, and in the WM of the parietal lobe point to decreased integrity of the superior longitudinal fasciculus. The alterations within the sagittal stratum may reflect age-related integrity loss of the communication between the frontal and occipital lobes via the fronto-occipital fasciculus (Schmahmann and Pandya, 2006).

Taken together, tissue integrity, as measured by FA, was decreased in the seventh relative to the third decade of life in local and commissural connections of the prefrontal cortex and in association fibers between the frontal lobe and other lobes. In addition to the frontal circuitry, increased age affected elements of the limbic system (cingulum, fornix, parahippocampal WM), parts of the corticospinal/ corticopontine tracts (corona radiata, internal capsule, midbrain $\mathrm{WM}$ ), and reciprocal connections between the medulla and the cerebellum (inferior cerebellar peduncle).

Possible microstructural underpinnings of the region-specific diffusivity patterns

The whole-WM comparison showed the expected patterns of agerelated increases in MD (Head et al., 2004; Pfefferbaum and Sullivan, 2003) and RD (Bhagat and Beaulieu, 2004; Ota et al., 2006; Sullivan et al., 2006a), and a less pronounced rise in AD (Bhagat and Beaulieu, 2004). In addition, the voxelwise TBSS analysis showed decreases in $\mathrm{MD}$ and $\mathrm{AD}$ in restricted regions showing a reduction in FA. The spatial distribution of age-related differences in $\mathrm{MD}, \mathrm{RD}$, and $\mathrm{AD}$ revealed five main patterns: (1) increase in $\mathrm{RD}, \mathrm{MD}$, and $\mathrm{AD}$; (2) an increase in $\mathrm{RD}$ only; (3) lowered FA without difference in other diffusivity parameters; (4) an increase in RD and a decrease in AD; and (5) a decrease in $\mathrm{AD}$ with a decrease in MD (Fig. 1 and Table 1 ).

In this study on healthy younger and older adults, there were no histological data available that would enable a more direct link between diffusivity patterns and region-specific microstructural alterations. Many previous histological-DTI studies have, however, investigated how different types of microstructural alterationsdemyelination, acute axonal injury, gliosis, Wallerian degeneration, chronic WM damage-translate into different patterns of differences in WM diffusivity properties. Guided by these studies, we can infer the possible neurobiological underpinnings of the observed patterns of age-related differences in diffusivity characteristics.

\section{Chronic WM degeneration (pattern 1)}

Within regions showing an age-related FA decrease, an increase in $\mathrm{RD}, \mathrm{MD}$, and partly also AD occupied the largest volume. Such a diffusivity pattern has been observed in the chronic stages of fiber degeneration after corpus callosotomy (Concha et al., 2006). After the transection of the corpus callosum, there is a decrease in $\mathrm{AD}$ as the fragmentation of axons creates barriers to the longitudinal displacement of water molecules. Next, the axonal membranes are degraded and the myelin sheaths fall apart, resulting in an increase in RD. The cellular debris is subsequently cleared by microglia, resulting in reestablishment or an increase in diffusion in the longitudinal direction (Concha et al., 2006; Sun et al., 2008).

Thus, this pattern of diffusivity differences likely reflects higher extracellular volume fraction and lower membrane density as a result of myelin and axon loss, a smaller volume fraction of axons, an increase in axonal spacing (Sen and Basser, 2005) as well as a reduction in extracellular tortuosity (Norris, 2001). The lack of decreased AD suggests that the cellular debris has been removed by phagocytic microglia, re-establishing diffusion parallel to the remaining axons.

Such diffusivity properties have been described in other cases of chronic WM damage. An increase in RD and MD and a decrease in FA were observed in amyotrophic lateral sclerosis (Cosottini et al., 2005). A decrease in FA together with a marked increase in MD reflects a loss of both axons and myelin in Balo's concentric sclerosis lesions (Lindquist et al., 2007) or in chronic stages of Wallerian degeneration after ischemic stroke (Thomalla et al., 2004). Decreased FA together with increased RD and AD was observed after traumatic WM injury in patients with unfavorable outcome, suggesting irreversible WM damage (Sidaros et al., 2008).

Interestingly, in our healthy older adults, we observed signatures of chronic WM degeneration mainly in the anterior parts of the corpus callosum and in the association fibers. Selective vulnerability of the association fibers has been linked to their relatively late myelination during development (Raz et al., 2000). As a result, most axons in these regions have not only a small diameter and thinner myelin sheaths but also a lower oligodendrocyte-to-axon ratio, making the glial cells more metabolically challenged. This should make the myelin sheaths more exposed to the potentially toxic extracellular environment and hence more vulnerable (Bartzokis, 2004; Bartzokis et al., 2004). This is in line with findings from a study on mono- and dizygotic twins, showing that anterior parts of the corpus callosum are more susceptible to environmental influences than its posterior parts (Pfefferbaum et al., 2001).

\section{Demyelination (pattern 2)}

FA reduction along with RD increase has been related to loss of myelin, as observed in acute demyelinating multiple sclerosis (Ciccarelli et al., 2006) and in animal studies of experimentally induced myelin loss (Song et al., 2002, 2005; Sun et al., 2006). Our results suggest that with increased age, some WM structures, such as the superior corona radiata (corticospinal and corticopontine tracts, superior thalamic radiations) and forceps major (posterior thalamic radiations/optic radiations), are affected primarily by demyelination, which are consistent with histological findings of an age-related loss of myelin and myelinated fibers in old age (Aboitiz et al., 1996; MeierRuge et al., 1992; Tang and Nyengaard, 1997; Terao et al., 1994).

\section{Subtle microstructural alterations (pattern 3)}

Reductions in FA alone, as observed in frontal gyri WM, suggest a mixture of subtle differences in RD and AD. Conceivably, this reflects mild microstructural alterations, such as minor fiber loss without gross tissue loss, leading to lowered FA but no net differences in MD (Rovaris et al., 2005; Sen and Basser, 2005). This loss of fiber coherence in the gyral WM could be connected to age-related thinning of cortical grey matter, known to affect particularly the frontal lobes (Salat et al., 2004).

\section{Secondary Wallerian degeneration (pattern 4)}

A decrease in FA with simultaneous increase in $\mathrm{RD}$, a decrease in $\mathrm{AD}$, and the resulting non-significant difference in MD has been observed in secondary Wallerian degeneration (Pierpaoli et al., 2001). This is considered to be a result of axonal loss, followed by increases in extracellular matrix and isotropic tissue structures, such as glia (Pierpaoli et al., 1996, 2001; Pierpaoli and Basser, 1996). This diffusivity pattern was present mainly in the retrolenticular part of the internal capsule, containing the optic radiations, as well as in the parahippocampal WM. Histological evidence suggests that there is considerable hippocampal gliosis in both demented and nondemented elderly adults (Beach et al., 1989). More importantly, WMHs typical for the healthy old population (Baloh and Vinters, 1995) may be the primary lesions from which Wallerian degeneration originates.

\section{Gliosis or early axonal injury (pattern 5)}

A decrease in FA accompanied by a decrease in AD and MD was present mainly in the parts of the corticospinal/corticopontine tracts at the level of the posterior limb of the internal capsule and in the 
cerebral peduncles in the midbrain. Lowered FA and AD (without RD or MD increases) has been reported in both rodents and humans in acute axonal damage involving axon swelling, fragmentation, and organelle accumulation (Concha et al., 2006; Song et al., 2003; Sun et al., 2006). Ischemic lesions may cause such acute axonal dysfunction. Another reason for reduced $\mathrm{AD}$ and $\mathrm{MD}$ is increased density of cellular membranes, for instance of glial cells, that restrict the freedom of diffusion (Beaulieu, 2002). This is in line with findings of an increased number of glia in late adulthood (Beach et al., 1989; Terry et al., 1987). Combining DTI with another imaging modality such as MR spectroscopy or magnetization transfer imaging could allow the discrimination between the acute axonal loss and glia infiltration in aging WM.

Diffusivity patterns in the seventh decade of life may reflect one time point of a complex cascade of age-related degeneration

Taken together, some WM regions seem to be in an advanced phase of injury in the seventh decade of life with possible loss of both axons and myelin. Some structures exhibit characteristics of a secondary Wallerian degeneration, whereas others may be affected by more subtle microstructural age alterations such as mere myelin loss, gliosis without tissue loss, and loss of axon coherence.

This cross-sectional study cannot address the temporal sequence or causal relations between differences in diffusion parameters. Nevertheless, it is tempting to speculate that some patterns may reflect earlier and other patterns later stages of progressing agerelated WM degeneration, at least in certain WM regions. For instance, in the genu of the corpus callosum, the predominant pattern points to chronic tissue loss, still some voxels indicate demyelination and Wallerian degeneration. Thus, it is conceivable that the focal demyelination or injury of the small diameter fibers of the corpus callosum develops into Wallerian degeneration, which in turn progresses to the chronic tissue loss. The pattern scheme introduced here should be extended to intermediate age groups (e.g., the 40-50 years age range) to identify patterns representing a cascade of degeneration and to longitudinal studies of WM changes in adulthood and old age.

\section{Limitations}

The described patterns of age-related diffusivity differences include four diffusivity measures. While RD and AD are by definition orthogonal, their composites, FA and MD, may not be orthogonal. Indeed, simultaneous age-related decreases in FA and increases in MD have been reported in some studies (for review, see Minati et al., 2007). To address this issue, we performed post-hoc analysis of FAMD correlations within specific patterns. This analysis showed that (a) the dependency between FA and MD is region-specific and (b) FA and MD are not necessarily correlated.

As predicted by the diffusivity patterns, in regions where a decrease in FA was accompanied by an increase in RD or AD, FA was negatively correlated with MD (genu of the corpus callosum, the superior corona radiata, and the forceps minor). Moreover, in the crus of the fornix, where increase in RD was counterbalanced by a decrease in $\mathrm{AD}, \mathrm{FA}$ was not related to $\mathrm{MD}$. In the cerebral peduncle, where FA decrease was observed together with a decrease in AD and MD, we observed a positive correlation between FA and MD, although not significant. This is in line with previous findings, that decreases in FA are not always accompanied by increases in MD (Pierpaoli et al., 2001). These results together with the literature indicate that MD and FA carry non-redundant information about WM microstructure. Although FA and MD may be highly correlated in certain types of microstructural alterations, it seems necessary to consider both of them to differentiate between region-specific aging mechanisms of WM. Similarly, adding $\mathrm{RD}$ and $\mathrm{AD}$ to the pattern analysis allows understanding which components of the diffusion tensor contributed to age differences in MD and FA, even if in some WM regions, these indices may show statistical dependence. For instance, FA can decrease as a result of an increase in RD or a decrease in AD. Conversely, by considering only $\mathrm{RD}$ and $\mathrm{AD}$ we would not know whether the observed decrease in $\mathrm{AD}$ is strong enough to significantly affect the shape of the diffusion ellipsoid (i.e., anisotropy) or the overall diffusivity within a voxel (MD).

Taken together, these results indicate that it is advantageous to consider all four measures when reporting age-related differences in diffusivity properties.

The age-related FA reductions in the fornix are present in figures of previous VBM studies, but they were not discussed (Camara et al., 2007; Hsu et al., 2008; Hugenschmidt et al., 2008; Salat et al., 2005), likely because there is a high risk of detecting a false positive due to suboptimal alignment of this fiber tract. Also, the body and column of the fornix have never been included in ROI analyses, presumably due to their small size and vicinity to the ventricles. In a recent TBSS study, Vernooij et al. (2008) showed that global age-related WM atrophy was related to decreased FA values in the fornix in adults over the age of 60 . This atrophy increases the partial-volume effect and may therefore explain the relatively high (in comparison to other WM structures) means, standard deviations, and the marked age-related increases in $\mathrm{RD}, \mathrm{AD}$, and MD observed in our study (Fig. S-1, Supplementary materials), indicative of partial-volume effects due to age-related atrophy in the body of the fornix (Sullivan et al., in press). Thus, even restricting the analysis to carefully sampled center-of-tract values in TBSS may not suffice to circumvent partial-volume effects in very thin structures such as fornix and external capsule. As the external capsule is tightly surrounded by grey matter structures, partial-volume effects should result in a decrease in MD (in addition to a decrease in FA), the opposite of what we observed. Hence, the observed increase in MD, RD, and $\mathrm{AD}$ likely reflects true fiber loss within the external capsule. In the fornix, however, microstructural mechanisms cannot be differentiated from macrostructural, atrophy-related differences.

Although by employing TBSS we sampled diffusivity values from the centres of the tracts, some voxels located on the WM skeleton were within the crossings of different fiber pathways. In these voxels, $\mathrm{RD}$ and $\mathrm{AD}$ cannot serve as markers of myelin and axon integrity, respectively (Song et al., 2002, 2003, 2005), and the patterns should be interpreted with caution. The diffusivity pattern in the genu of the corpus callosum and the anterior limb of the internal capsule suggests chronic fiber loss. Major parts of the corpus callosum splenium showed no decrease in FA, and the posterior limb of the internal capsule may be affected primarily by gliosis and axonal insult. The fact that WM regions with compact and coherent fibers differed in their age-related diffusivity patterns confirms the validity of the pattern approach adopted here.

Comparing groups of younger and older adults makes it impossible to assess possible nonlinearities in WM microstructure differences between the 3rd and 7th decades of life. At present, evidence in favor of such nonlinearities in WM volume rests on a small number of crosssectional studies (Courchesne et al., 2000; Ge et al., 2002). In a crosssectional DTI study with continuous age sampling, diffusivity indices for all major WM tracts were well approximated by linear functions (Sullivan et al., in press). Clearly, longitudinal data on sufficiently large samples are needed to verify the onset and shape of average age trends, the degree of between-person differences around these trends, and the cascade of senescent changes in WM integrity proposed in this article.

\section{Summary and outlook}

We used TBSS to assess age-related differences in WM diffusivity properties in multiple sites within the entire cerebral WM while accounting for morphological alterations in older adults. Because we 
used a whole-brain approach that is sensitive to small alterations in WM integrity, we were able to detect reductions in FA in regions that have rarely been considered in ROI-based studies, such as the parahippocampal WM. We found that the overall pattern of agerelated loss of WM integrity goes beyond the common notion of a simple anteroposterior gradient of FA decline. Still, the pattern of lowered FA suggests primary disruption of the connections of the frontal lobe. Within the regions exhibiting reduced FA, higher RD may represent age-related decreases in myelin content and integrity. The pattern of simultaneous increases in RD and MD, likely indicative of chronic WM degeneration, matches the notion that the late myelinating association fibers are most susceptible to accumulating age-related injuries. Reductions in FA only may be the result of early or subtle pathological processes. A decline in FA and AD with an increase in RD likely represents secondary Wallerian degeneration, whereas a decrease in AD and MD may be a manifestation of glial infiltration. This is the first description of regional differences in all main diffusivity parameters, suggesting differential age-related mechanisms of integrity loss within cerebral WM. We also showed that age-related microstructural alterations lead to increased dependency between RD and $A D$, which are by definition orthogonal (see Supplementary materials). This increase in RD-AD correlation is likely connected to increased isotropic volume fraction in aging WM, regardless of the underlying pathological process. Importantly, this study reinforces the necessity of relating the different diffusivity indices directly to each other to discriminate between different microstructural mechanisms which are indistinguishable if characterized by FA or MD alone.

Behaviorally, we observed the typical pattern of healthy normal aging, with an age-related deficit in a marker test of fluid intelligence and age-related increase in a marker of crystallized intelligence. Therefore, it is unlikely that the observed mean age group differences in white matter integrity were a gross misrepresentation of the differences associated with normal aging. Conceptually, we proposed a refined scheme for the interpretation of age-associated differences in diffusivity parameters on a voxel level. In future studies, the diffusivity pattern scheme should be extended to the analysis of adolescents and middle-aged adults. Further, longitudinal data are needed to examine individual differences in diffusivity changes across the adult life span and their relations to individual differences in behavioral change.

\section{Acknowledgments}

This research was supported by the Innovation Fund of the Max Planck Society (M.FE.A.BILD0005) and the German Federal Ministry for Research to the Berlin NeuroImaging Center (01G00501), the Swedish Research Council (521-2007-2892 to L.B.), and Swedish Brain Power (to L.B.). A.Z.B. received a predoctoral fellowship from the International Max Planck Research School, The Life Course: Evolutionary and Ontogenetic Dynamics (LIFE).

The authors thank Nils Bodammer for valuable comments, and Peter Brunecker, Irene Nagel and the student assistants for their assistance in data collection.

The authors declare no conflicts of interests.

\section{Appendix A. Supplementary data}

Supplementary data associated with this article can be found, in the online version, at doi:10.1016/j.neuroimage.2009.09.041.

\section{References}

Abe, O., Aoki, S., Hayashi, N., Yamada, H., Kunimatsu, A., Mori, H., Yoshikawa, T., Okubo, T., Ohtomo, K., 2002. Normal aging in the central nervous system: quantitative MR diffusion-tensor analysis. Neurobiol. Aging 23, 433-441.
Aboitiz, F., Rodriguez, E., Olivares, R., Zaidel, E., 1996. Age-related changes in fibre composition of the human corpus callosum: sex differences. NeuroReport 7, 1761-1764.

Ardekani, S., Kumar, A., Bartzokis, G., Sinha, U., 2007. Exploratory voxel-based analysis of diffusion indices and hemispheric asymmetry in normal aging. Magn. Reson. Imaging 25, 154-167.

Assaf, Y., Pasternak, O., 2008. Diffusion tensor imaging (DTI)-based white matter mapping in brain research: a review. J. Mol. Neurosci. 34, 51-61.

Baloh, R.W., Vinters, H.V., 1995. White matter lesions and disequilibrium in older people: II. Clinicopathologic correlation. Arch. Neurol. 52, 975-981.

Bartzokis, G., 2004. Age-related myelin breakdown: a developmental model of cognitive decline and Alzheimer's disease. Neurobiol. Aging 25, 5-18.

Bartzokis, G., Sultzer, D., Lu, P.H., Nuechterlein, K.H., Mintz, J., Cummings, J.L., 2004. Heterogeneous age-related breakdown of white matter structural integrity: implications for cortical "disconnection" in aging and Alzheimer's disease. Neurobiol. Aging 25, 843-851.

Basser, P.J., 1995. Inferring microstructural features and the physiological state of tissues from diffusion-weighted images. NMR Biomed. 8, 333-344.

Basser, P.J., Pierpaoli, C., 1996. Microstructural and physiological features of tissues elucidated by quantitative-diffusion-tensor MRI. J. Magn. Reson., B 111, 209-219.

Basser, P.J., Pierpaoli, C., 1998. A simplified method to measure the diffusion tensor from seven MR images. Magn. Reson. Med. 39, 928-934.

Beach, T.G., Walker, R., McGeer, E.G., 1989. Patterns of gliosis in Alzheimer's disease and aging cerebrum. Glia 2, 420-436.

Beaulieu, C., 2002. The basis of anisotropic water diffusion in the nervous system-a technical review. NMR Biomed. 15, 435-455.

Beaulieu, C., Does, M.D., Snyder, R.E., Allen, P.S., 1996. Changes in water diffusion due to Wallerian degeneration in peripheral nerve. Magn. Reson. Med. 36, 627-631.

Bhagat, Y.A., Beaulieu, C., 2004. Diffusion anisotropy in subcortical white matter and cortical gray matter: changes with aging and the role of CSF-suppression. J. Magn. Reson. Imaging 20, 216-227.

Bucur, B., Madden, D.J., Spaniol, J., Provenzale, J.M., Cabeza, R., White, L.E., Huettel, S.A., 2008. Age-related slowing of memory retrieval: contributions of perceptual speed and cerebral white matter integrity. Neurobiol. Aging 29, 1070-1079.

Cabeza, R., Daselaar, S.M., Dolcos, F., Prince, S.E., Budde, M., Nyberg, L., 2004. Taskindependent and task-specific age effects on brain activity during working memory, visual attention and episodic retrieval. Cereb. Cortex 14, 364-375.

Camara, E., Bodammer, N., Rodriguez-Fornells, A., Tempelmann, C., 2007. Age-related water diffusion changes in human brain: a voxel-based approach. NeuroImage 34, 1588-1599.

Ciccarelli, O., Behrens, T.E., Altmann, D.R., Orrell, R.W., Howard, R.S., Johansen-Berg, H., Miller, D.H., Matthews, P.M., Thompson, A.J., 2006. Probabilistic diffusion tractography: a potential tool to assess the rate of disease progression in amyotrophic lateral sclerosis. Brain 129, 1859-1871.

Concha, L., Gross, D.W., Wheatley, B.M., Beaulieu, C., 2006. Diffusion tensor imaging of time-dependent axonal and myelin degradation after corpus callosotomy in epilepsy patients. NeuroImage 32, 1090-1099.

Cosottini, M., Giannelli, M., Siciliano, G., Lazzarotti, G., Nichelassi, M.C., Del Corona, A., Bartolozzi, C., Murri, L., 2005. Diffusion-tensor MR imaging of corticospinal tract in amyotrophic lateral sclerosis and progressive muscular atrophy. Radiology 237, 258-264.

Courchesne, E., Chisum, H.J., Townsend, J., Cowles, A., Covington, J., Egaas, B., Harwood, M., Hinds, S., Press, G.A., 2000. Normal brain development and aging: quantitative analysis at in vivo MR imaging in healthy volunteers. Radiology 216, 672-682.

Damoiseaux, J.S., Smith, S.M., Witter, M.P., Arigita, E.J., Barkhof, F., Scheltens, P., Stam, C.J., Zarei, M., Rombouts, S.A., 2009. White matter tract integrity in aging and Alzheimer's disease. Hum. Brain Mapp. 30, 1051-1059.

Daselaar, S.M., Fleck, M.S., Dobbins, I.G., Madden, D.J., Cabeza, R., 2006. Effects of healthy aging on hippocampal and rhinal memory functions: an event-related fMRI study. Cereb. Cortex 16, 1771-1782.

Eichenbaum, H., Cohen, N.J., 2001. From Conditioning to Conscious Recollection: Memory Systems of the Brain, 1st ed. Oxford Univ. Press Inc, USA.

Ge, Y., Grossman, R.I., Babb, J.S., Rabin, M.L., Mannon, L.J., Kolson, D.L., 2002. Age-related total gray matter and white matter changes in normal adult brain: Part I. Volumetric MR imaging analysis. Am. J. Neuroradiol. 23, 1327-1333.

Giedd, J., 1999. Brain development: IX. Human brain growth. Am. J. Psychiatry 156, 4.

Grieve, S.M., Williams, L.M., Paul, R.H., Clark, C.R., Gordon, E., 2007. Cognitive aging, executive function, and fractional anisotropy: a diffusion tensor MR imaging study. Am. J. Neuroradiol. 28, 226-235.

Head, D., Buckner, R.L., Shimony, J.S., Williams, L.E., Akbudak, E., Conturo, T.E., McAvoy, M., Morris, J.C., Snyder, A.Z., 2004. Differential vulnerability of anterior white matter in nondemented aging with minimal acceleration in dementia of the Alzheimer type: evidence from diffusion tensor imaging. Cereb. Cortex 14, 410-423.

Hofer, S., Frahm, J., 2006. Topography of the human corpus callosum revisitedcomprehensive fiber tractography using diffusion tensor magnetic resonance imaging. Neurolmage 32, 989-994.

Hsu, J.L., Leemans, A., Bai, C.H., Lee, C.H., Tsai, Y.F., Chiu, H.C., Chen, W.H., 2008. Gender differences and age-related white matter changes of the human brain: a diffusion tensor imaging study. NeuroImage 39, 566-577.

Hugenschmidt, C.E., Peiffer, A.M., Kraft, R.A., Casanova, R., Deibler, A.R., Burdette, J.H., Maldjian, J.A., Laurienti, P.J., 2008. Relating imaging indices of white matter integrity and volume in healthy older adults. Cereb. Cortex 18, 433-442.

Jones, D.K., Symms, M.R., Cercignani, M., Howard, R.J., 2005. The effect of filter size on VBM analyses of DT-MRI data. NeuroImage 26, 546-554.

Li, S.C., Lindenberger, U., Hommel, B., Aschersleben, G., Prinz, W., Baltes, P.B., 2004. Transformations in the couplings among intellectual abilities and constituent cognitive processes across the life span. Psychol. Sci. 15, 155-163. 
Lindquist, S., Bodammer, N., Kaufmann, J., Konig, F., Heinze, H.J., Bruck, W., Sailer, M. 2007. Histopathology and serial, multimodal magnetic resonance imaging in a multiple sclerosis variant. Mult. Scler. 13, 471-482.

Madden, D.J., Whiting, W.L., Huettel, S.A., White, L.E., MacFall, J.R., Provenzale, J.M., 2004. Diffusion tensor imaging of adult age differences in cerebral white matter: relation to response time. Neurolmage 21, 1174-1181.

Marner, L., Nyengaard, J.R., Tang, Y., Pakkenberg, B., 2003. Marked loss of myelinated nerve fibers in the human brain with age. J. Comp. Neurol. 462, 144-152.

Medina, D., DeToledo-Morrell, L., Urresta, F., Gabrieli, J.D., Moseley, M., Fleischman, D., Bennett, D.A., Leurgans, S., Turner, D.A., Stebbins, G.T., 2006. White matter changes in mild cognitive impairment and AD: a diffusion tensor imaging study. Neurobiol. Aging 27, 663-672.

Meier-Ruge, W., Ulrich, J., Bruhlmann, M., Meier, E., 1992. Age-related white matter atrophy in the human brain. Ann. N. Y. Acad. Sci. 673, 260-269.

Minati, L., Grisoli, M., Buzzone, M.G., 2007. MR spectroscopy, functional MRI, and diffusion-tensor imaging in the aging brain: a conceptual review. J. Geriatr. Psychiatry Neurol. 20, 3-21.

Nichols, T.E., Holmes, A.P., 2002. Nonparametric permutation tests for functional neuroimaging: a primer with examples. Hum. Brain Mapp. 15, 1-25.

Norris, D.G., 2001. The effects of microscopic tissue parameters on the diffusion weighted magnetic resonance imaging experiment. NMR Biomed. 14, 77-93.

Ota, M., Obata, T., Akine, Y., Ito, H., Ikehira, H., Asada, T., Suhara, T., 2006. Age-related degeneration of corpus callosum measured with diffusion tensor imaging. Neurolmage 31, 1445-1452.

Paxton, J.L., Barch, D.M., Racine, C.A., Braver, T.S., 2008. Cognitive control, goal maintenance, and prefrontal function in healthy aging. Cereb. Cortex 18, 1010-1028.

Persson, J., Lind, J., Larsson, A., Ingvar, M., Cruts, M., Van Broeckhoven, C., Adolfsson, R., Nilsson, L.G., Nyberg, L., 2006. Altered brain white matter integrity in healthy carriers of the APOE epsilon4 allele: a risk for AD? Neurology 66, 1029-1033.

Pfefferbaum, A., Sullivan, E.V., 2003. Increased brain white matter diffusivity in normal adult aging: relationship to anisotropy and partial voluming. Magn. Reson. Med. 49, 953-961.

Pfefferbaum, A., Sullivan, E.V., Hedehus, M., Adalsteinsson, E., Lim, K.O., Moseley, M., 2000a. In vivo detection and functional correlates of white matter microstructural disruption in chronic alcoholism. Alcohol., Clin. Exp. Res. 24, 1214-1221.

Pfefferbaum, A., Sullivan, E.V., Hedehus, M., Lim, K.O., Adalsteinsson, E., Moseley, M., 2000b. Age-related decline in brain white matter anisotropy measured with spatially corrected echo-planar diffusion tensor imaging. Magn. Reson. Med. 44, 259-268.

Pfefferbaum, A., Sullivan, E.V., Carmelli, D., 2001. Genetic regulation of regional microstructure of the corpus callosum in late life. NeuroReport 12, 1677-1681.

Pfefferbaum, A., Adalsteinsson, E., Sullivan, E.V., 2005. Frontal circuitry degradation marks healthy adult aging: evidence from diffusion tensor imaging. Neurolmage 26, 891-899.

Pierpaoli, C., Basser, P.J., 1996. Toward a quantitative assessment of diffusion anisotropy. Magn. Reson. Med. 36, 893-906.

Pierpaoli, C., Jezzard, P., Basser, P.J., Barnett, A., Di Chiro, G., 1996. Diffusion tensor MR imaging of the human brain. Radiology 201, 637-648.

Pierpaoli, C., Barnett, A., Pajevic, S., Chen, R., Penix, L.R., Virta, A., Basser, P., 2001. Water diffusion changes in Wallerian degeneration and their dependence on white matter architecture. NeuroImage 13, 1174-1185.

Raz, N., Rodrigue, K.M., 2006. Differential aging of the brain: patterns, cognitive correlates and modifiers. Neurosci. Biobehav. Rev. 30, 730-748.

Raz, N., Williamson, A., Gunning-Dixon, F., Head, D., Acker, J.D., 2000. Neuroanatomical and cognitive correlates of adult age differences in acquisition of a perceptualmotor skill. Microsc. Res. Tech. 51, 85-93.

Raz, N., Lindenberger, U., Rodrigue, K.M., Kennedy, K.M., Head, D., Williamson, A. Dahle, C., Gerstorf, D., Acker, J.D., 2005. Regional brain changes in aging healthy adults: general trends, individual differences and modifiers. Cereb. Cortex 15, $1676-1689$.

Reese, T.G., Heid, O., Weisskoff, R.M., Wedeen, V.J., 2003. Reduction of eddy-currentinduced distortion in diffusion MRI using a twice-refocused spin echo. Magn. Reson. Med. 49, 177-182.

Rovaris, M., Gass, A., Bammer, R., Hickman, S.J., Ciccarelli, O., Miller, D.H., Filippi, M., 2005. Diffusion MRI in multiple sclerosis. Neurology 65, 1526-1532.

Rueckert, D., Sonoda, L.I., Hayes, C., Hill, D.L., Leach, M.O., Hawkes, D.J., 1999. Nonrigid registration using free-form deformations: application to breast MR images. IEEE Trans. Med. Imaging 18, 712-721.

Salat, D.H., Buckner, R.L., Snyder, A.Z., Greve, D.N., Desikan, R.S., Busa, E., Morris, J.C., Dale, A.M., Fischl, B., 2004. Thinning of the cerebral cortex in aging. Cereb. Cortex $14,721-730$.

Salat, D.H., Tuch, D.S., Greve, D.N., van der Kouwe, A.J., Hevelone, N.D., Zaleta, A.K., Rosen, B.R., Fischl, B., Corkin, S., Rosas, H.D., Dale, A.M., 2005. Age-related alterations in white matter microstructure measured by diffusion tensor imaging. Neurobiol. Aging 26, 1215-1227.

Schmahmann, J.D., Pandya, D.N., 2006. Fiber Pathways of the Brain, 1st ed. Oxford Univ. Press, Oxford.

Sen, P.N., Basser, P.J., 2005. A model for diffusion in white matter in the brain. Biophys. J. $89,2927-2938$.
Sidaros, A., Engberg, A.W., Sidaros, K., Liptrot, M.G., Herning, M., Petersen, P., Paulson, O.B., Jernigan, T.L., Rostrup, E., 2008. Diffusion tensor imaging during recovery from severe traumatic brain injury and relation to clinical outcome: a longitudinal study. Brain 131, 559-572.

Smith, S.M., 2002. Fast robust automated brain extraction. Hum. Brain Mapp. 17, 143-155.

Smith, S.M., Jenkinson, M., Woolrich, M.W., Beckmann, C.F., Behrens, T.E., JohansenBerg. H., Bannister, P.R., De Luca, M., Drobnjak, I., Flitney, D.E., Niazy, R.K., Saunders, J., Vickers, J., Zhang, Y., De Stefano, N., Brady, J.M., Matthews, P.M., 2004. Advances in functional and structural MR image analysis and implementation as FSL NeuroImage 23 (Suppl. 1), S208-S219.

Smith, S.M., Jenkinson, M., Johansen-Berg, H., Rueckert, D., Nichols, T.E., Mackay, C.E., Watkins, K.E., Ciccarelli, O., Cader, M.Z., Matthews, P.M., Behrens, T.E.J., 2006 Tract-based spatial statistics: voxelwise analysis of multi-subject diffusion data. NeuroImage 31, 1487-1505.

Smith, S.M., Johansen-Berg, H., Jenkinson, M., Rueckert, D., Nichols, T.E., Miller, K.L., Robson, M.D., Jones, D.K., Klein, J.C., Bartsch, A.J., Behrens, T.E., 2007. Acquisition and voxelwise analysis of multi-subject diffusion data with tract-based spatial statistics. Nat. Protoc. 2, 499-503.

Song, S.K., Sun, S.W., Ramsbottom, M.J., Chang, C., Russell, J., Cross, A.H., 2002. Dysmyelination revealed through MRI as increased radial (but unchanged axial) diffusion of water. NeuroImage 17, 1429-1436.

Song, S.K., Sun, S.W., Ju, W.K., Lin, S.J., Cross, A.H., Neufeld, A.H., 2003. Diffusion tensor imaging detects and differentiates axon and myelin degeneration in mouse optic nerve after retinal ischemia. Neurolmage 20, 1714-1722.

Song, S.K., Yoshino, J., Le, T.Q., Lin, S.J., Sun, S.W., Cross, A.H., Armstrong, R.C., 2005 Demyelination increases radial diffusivity in corpus callosum of mouse brain. NeuroImage 26, 132-140.

Stadlbauer, A., Salomonowitz, E., Strunk, G., Hammen, T., Ganslandt, O., 2008 Quantitative diffusion tensor fiber tracking of age-related changes in the limbic system. Eur. Radiol. 18, 130-137.

Sullivan, E.V., Pfefferbaum, A., 2006. Diffusion tensor imaging and aging. Neurosci. Biobehav. Rev. 30, 749-761.

Sullivan, E.V., Adalsteinsson, E., Hedehus, M., Ju, C., Moseley, M., Lim, K.O., Pfefferbaum, A., 2001. Equivalent disruption of regional white matter microstructure in ageing healthy men and women. NeuroReport 12, 99-104.

Sullivan, E.V., Adalsteinsson, E., Pfefferbaum, A., 2006a. Selective age-related degradation of anterior callosal fiber bundles quantified in vivo with fiber tracking. Cereb. Cortex 16, 1030-1039.

Sullivan, E.V., Adalsteinsson, E., Sood, R., Mayer, D., Bell, R., McBride, W., Li, T.K. Pfefferbaum, A., 2006b. Longitudinal brain magnetic resonance imaging study of the alcohol-preferring rat: Part I. Adult brain growth. Alcohol., Clin. Exp. Res. 30 $1234-1247$.

Sullivan, E.V., Rohlfing, T., Pfefferbaum, A., in press. Quantitative fiber tracking of latera and interhemispheric white matter systems in normal aging: relations to timed performance. Neurobiol. Aging. doi:10.1016/j.neurobiolaging.2008.04.007.

Sun, S.W., Liang, H.F., Trinkaus, K., Cross, A.H., Armstrong, R.C., Song, S.K., 2006 Noninvasive detection of cuprizone induced axonal damage and demyelination in the mouse corpus callosum. Magn. Reson. Med. 55, 302-308.

Sun, S.W., Liang, H.F., Cross, A.H., Song, S.K., 2008. Evolving Wallerian degeneration after transient retinal ischemia in mice characterized by diffusion tensor imaging. Neurolmage 40, 1-10.

Tang, Y., Nyengaard, J.R., 1997. A stereological method for estimating the total length and size of myelin fibers in human brain white matter. J. Neurosci. Methods 73, 193-200.

Terao, S., Sobue, G., Hashizume, Y., Shimada, N., Mitsuma, T., 1994. Age-related changes of the myelinated fibers in the human corticospinal tract: a quantitative analysis Acta Neuropathol. 88, 137-142.

Terry, R.D., DeTeresa, R., Hansen, L.A., 1987. Neocortical cell counts in normal human adult aging. Ann. Neurol. 21, 530-539.

Thomalla, G., Glauche, V., Koch, M.A., Beaulieu, C., Weiller, C., Rother, J., 2004. Diffusion tensor imaging detects early Wallerian degeneration of the pyramidal tract after ischemic stroke. Neurolmage 22, 1767-1774.

Vernooij, M.W., de Groot, M., van der Lugt, A., Ikram, M.A., Krestin, G.P., Hofman, A., Niessen, W.J., Breteler, M.M., 2008. White matter atrophy and lesion formation explain the loss of structural integrity of white matter in aging. NeuroImage 43, $470-477$.

Wahlund, L.O., Barkhof, F., Fazekas, F., Bronge, L., Augustin, M., Sjogren, M., Wallin, A. Ader, H., Leys, D., Pantoni, L., Pasquier, F., Erkinjuntti, T., Scheltens, P., 2001. A new rating scale for age-related white matter changes applicable to MRI and CT. Stroke $32,1318-1322$.

Yoon, B., Shim, Y.S., Lee, K.S., Shon, Y.M., Yang, D.W., 2008. Region-specific changes of cerebral white matter during normal aging: a diffusion-tensor analysis. Arch. Gerontol. Geriatr. 47, 129-138.

Zhang, Y., Brady, M., Smith, S., 2001. Segmentation of brain MR images through a hidden Markov random field model and the expectation maximization algorithm. IEEE Trans. Med. Imag. 20, 45-57.

Zhang, Y., Du, A.T., Hayasaka, S., Jahng, G.H., Hlavin, J., Zhan, W., Weiner, M.W. Schuff, N., in press. Patterns of age-related water diffusion changes in human brain by concordance and discordance analysis. Neurobiol. Aging. doi:10.1016/j. neurobiolaging.2008.10.009. 